\title{
Using a Species Distribution Model to guide NSW surveys of the Long-footed Potoroo (Potorous longipes)
}

Mareshell Wauchope-Drumm ${ }^{1}$, Joss Bentley ${ }^{2}$, Linda J Beaumont ${ }^{1}$, John B Baumgartner $^{1}$, David A Nipperess ${ }^{1}$

1 Department of Biological Sciences, Macquarie University, NSW, 2109, Australia

2 Office of Environment and Heritage, New South Wales Government, Queanbeyan, NSW, 2620, Australia

Corresponding Author: Mareshell Wauchope-Drumm

Department of Biological Sciences, Macquarie University, NSW, 2109, Australia

Email: mareshell.wauchope-drumm@mq.edu.au

Mobile: 0435551485

\section{Acknowledgements}

This project was conducted as part of a Master of Research (MRES) undertaken by Mareshell Wauchope-Drumm at Macquarie University. The Department of Biology contributed to the cost of fieldwork. The Office of Environment and Heritage (OEH) funded Joss Bentley and provided fieldwork equipment. Franz Peters and Rachel Butterworth of NSW National Parks and Wildlife Service are thanked for their invaluable guidance and support in the field.

All survey procedures were approved by OEH Animal Research Authority (approval number 150804/01) and Macquarie University Animal Ethics Committee (approval number 5201600599 - Collaboration (OEH 150804/01)).

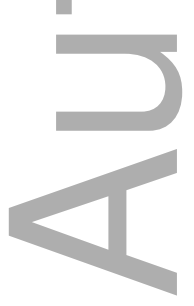

This is the author manuscript accepted for publication and has undergone full peer review but has not been through the copyediting, typesetting, pagination and proofreading process, which may lead to differences between this version and the Version of Record. Please cite this article as doi: $10.1111 /$ AEC.12804

This article is protected by copyright. All rights reserved 
MRS. MARESHELL WAUCHOPE-DRUMM (Orcid ID : 0000-0002-1414-8925)

Article type

: Original Article

Using a Species Distribution Model to guide NSW surveys of the Long-footed Potoroo (Potorous longipes)

Abstract

11 Knowledge of threatened species' distributions is essential for effective conservation decision-making. Species distribution models (SDMs) are widely used to map species' geographic ranges, identify new areas of suitable habitat and guide field surveys. In New South Wales (NSW), Australia, there are grave doubts about whether populations of the Critically Endangered long-footed potoroo (Potorous longipes) remain extant, and identification of occupied sites is a high priority for its conservation. We used an SDM (Maxent) to identify regions in NSW that may have suitable habitat for the potoroo. The SDM was built with seven climate layers and had strong predictive performance (crossvalidated $A U C=0.94)$. We then combined this information on habitat suitability with vegetation and topography, to identify 58 survey sites across NSW. From April 2016-May 2017, we undertook six field trips deploying six to eight cameras at each site for 52-63 days, resulting in 25,120 camera trap nights. A total of 215,759 images captured 43 native and feral animal species, but no long-footed potoroos. Following the survey, newly available, independent presence and absence data were used to validate our model. A Kruskal-Wallis $\mathrm{H}$ Test indicated that habitat suitability values were significantly higher at presence locations than absence locations $(H=58.66, D F=1, P<0.001)$. Finally, we refitted the Maxent model with the new data, and identified additional regions that future surveys could explore. We conclude, however, that if the long-footed potoroo remains extant in NSW, it is extremely rare.

Keywords: Species distribution model, Maxent, conservation biology, presence-only model, threatened species. 


\section{INTRODUCTION}

Surveys for rare and threatened species are essential for monitoring populations and supporting conservation outcomes. However, knowing where to direct survey effort is often hampered by a paucity of data regarding species' distributions, biology and ecology. Species distribution models (SDMs) provide an approach to mapping potentially suitable habitat to guide field surveys (Aizpurua et al. 2015) and have been used in this manner for a variety of taxa, including marine invertebrates (Rooper et al., 2016), amphibians (Groff et al. 2014), reptiles (Stratmann et al. 2016), birds (Aizpurua et al. 2015) and mammals (Lumsden et al. 2013). The application of SDMs is appealing as it is efficient (e.g. relative to random sampling, Guisan et al. 2006) and research has demonstrated that the detection of rare species is greatly improved when surveys are guided by SDMs (Guisan et al. 2006, Le Lay et al. 2010).

The long-footed potoroo (Potorous longipes) is a small marsupial rat kangaroo (1.5-2.3 kg) (Scotts \& Seebeck 1989) listed as Endangered in Australia (Environment Protection and Biodiversity Conservation Act 1999). Rare and elusive, it was described as a separate species in 1980, having previously been considered to be the more broadly distributed, vulnerable, long-nosed potoroo ( $P$. tridactylus) (Seebeck \& Johnston 1980). Since then, three small, disjunct populations have been located: two in north-eastern Victoria (Department of Sustainability and Environment (DSE) 2009), and one in south-eastern New South Wales (NSW) (National Parks and Wildlife Service (NPWS) 2002). The recently updated IUCN listing estimates that no more than a few thousand individuals remain (Woinarski \& Burbidge 2016).

The long-footed potoroo has been found across various ecological vegetation classes, altitudes (150-1370m), topographic types (from creeks to ridge tops) and forest age classes (e.g. eight-year regrowth post-timber harvesting to old growth forests) (Scotts \& Seeback 1989; Green et al. 1998; Chick et al. 2006; Elsner et al. 2012). Its home range is estimated to be 14-100ha (Green et al. 1998; DSE 2009), with preferred areas having moist soils and sheltered aspects, likely due to the species' relationship with fungal food sources (DSE 2009; NPWS 2002). Indeed, the long-footed potoroo is the most fungidependent mammal in Australia: on average, $91 \%$ of its diet comprises truffle-like fungi, with the remainder consisting of invertebrates and plants (Green et al. 1999). As such, year-round fungal abundance and diversity is likely a key requirement for the distribution of this species (NPWS 2002). 
All records of long-footed potoroos in NSW occur within the boundaries of the South-East

66 Forests National Park (SEFNP) (Figure 2). Multiple intensive surveys have been 67 undertaken in NSW to evaluate the species' distribution and patterns of occupancy. Hair 68 tubing and scat surveys in 1986 and 1989 confirmed the presence of the species within 69 the Sheep Station Creek catchment of SEFNP (Claridge 2002; NPWS 2002). These records, together with records from Victoria, were used to calibrate a BIOCLIM SDM, the results of which informed extensive live-trap and hair tube surveys conducted between 1990-1995 (Claridge 2002, Nix 1986) The surveys, however, yielded fewer than seventeen definitive records, with no live captures or direct observations made (Claridge 2002, NPWS 2002). The most recent survey undertaken in 2008/09 used scat searches, hair tubes and low-intensity camera trap surveys and yielded no new findings (unpublished data, J. Bentley).

In Victoria, however, recent camera surveys for the long-footed potoroo have had considerably more success. A multi-year survey program (2011-2016) detected individuals at 13 sites (pers. comm. Marc Perri, DELWP 21 February 2017), including a new population in the Gippsland area west of the Snowy River (Lumsden et al. 2013). In a separate study, predictions from an SDM guided a 2012 survey for the species in Gippsland, with long-footed potoroos detected at 41 sites (Lumsden et al. 2013). Of these, $12 \%$ were outside the previous known range of this species (Lumsden et al. 2013). In addition to the extension of surveys into new areas, other factors may have contributed to increased detection of the potoroo, including an increase in potoroo numbers resulting from improvements in predator control (fox baiting) and habitat recovery following the Millennium Drought (2002-2010). Most recently, camera surveys undertaken in 2016, as part of the Southern Ark Program (monitoring fox control) in Victoria, gave rise to 67 new detections across 35 locations (pers. comm. Andy Murray, DELWP, 1 December 2016).

In contrast, the failure of additional surveys to detect the species in parts of NSW where it was previously found raises concerns about possible extinction in the state. These concerns are echoed in the decision to upgrade the species' conservation status in NSW to Critically Endangered (NSW Scientific Committee (NSW SC) 2015). Alternatively, these findings might suggest: 1) surveys did not reliably census the species in this region, 2) the long-footed potoroo is present at extremely low densities, or 3) sampling occurred in the wrong locations.

97 Since the original BIOCLIM model was constructed, the species has been detected in new areas in Victoria, expanding the long-footed potoroo's distribution and resulting in the 
discovery of populations in new habitat types, e.g. drier coastal (Cape Conran) and hinterland habitat (Lumsden et al. 2013; Elsner et al. 2012). Furthermore, additional environmental datasets have become available, and SDM techniques (e.g., Maxent: Phillips et al. 2006; Elith et al. 2006; Merow et al. 2013) have advanced considerably. Given these advances, our objective was to model suitable habitat for the long-footed potoroo, and use this updated SDM to guide camera surveys aimed at locating extant populations in NSW. Further, in the absence of detailed data on abundance or other proxy measures of long-footed potoroo habitat quality in NSW, this study proposed to use field surveys to ground-truth the SDM predictions. Such information is critical to assess the likelihood that populations of this marsupial remain extant in NSW and, if so, aid the

\section{METHODS}

\section{Species Distribution Modelling - Maxent}

112 To establish the long-footed potoroo's current potential distribution in NSW, species distribution modelling was undertaken with Maxent (Version 3.3.3k) (Phillips et al. 2006; Phillips \& Dudík 2008). This type of SDM does not require absence data and is wellrecognised as having high predictive performance (Elith et al. 2006). The program requires up to three key data inputs: i) species occurrence data; ii) data describing environmental predictor variables across the study area; and iii) "background" points representing a sample of the available environment across the study area (optionally adjusted for sampling bias) (Syfert et al. 2013). To investigate the impact of different covariate combinations on model predictions, several model iterations were run using Maxent's default settings, with two exceptions relating to background points and feature selection (outlined below). The Maxent models in this paper were calibrated at $250 \times 250 \mathrm{~m}$ resolution, thought to be appropriate for capturing spatial environmental heterogeneity relevant to potoroo habitat, and permitting the use of a range of available climate, vegetation and topographic data.

\section{Occurrence Data}

127 Species occurrence records were obtained from the Atlas of Living Australia (ALA) 128 database (http://www.ala.org.au). The 531 occurrence records were examined to identify 129 definitive records of the species (i.e. locations where the animals were live-trapped, 130 camera-trapped, detected by hair-tubes; and/or had reliable expert observers). Spatially 131 invalid or duplicate records, and those that were poorly georeferenced (e.g. coordinate uncertainty $>1000 \mathrm{~m}$ ), were deleted. As the potoroo was identified as a distinct species in This article is protected by copyright. All rights reserved 
1980, records with dates stated as 'first of the century' (i.e.1/1/1900) or before 1980 were included only if they were sourced from a "preserved specimen". Following the data cleaning process, there were 341 occurrence records remaining; 34 from NSW and 307 from Victoria. The occurrence records were projected to the Australian Albers Equal Area (EPSG:3577) coordinate system and reduced to one record per grid cell (250m resolution) in the R statistical computing environment (v3.1.1) (R Development Core Team, 2014), leaving a total of 272 presence points for use in the model.

\section{Environmental Variables}

We used climate data for the period 1990-2009 that was generated as part of the NSW and ACT Regional Climate Modelling (NARCliM) project (Evans et al. 2014). These data represent a set of 35 bioclimatic variables, developed at a spatial resolution of 9 arcseconds (Hutchinson \& Xu 2015; Appendix S1). Using a cubic spline, we reprojected the data to EPSG:3577 at a resolution of $250 \times 250 \mathrm{~m}$. Slope and aspect (both measured in degrees) were calculated using the gdaldem function implemented in the gdalUtils $R$ package (Greenberg \& Mattiuzzi, 2015). Aspect was decomposed to two variables expressing the north-south (northness; cosine of aspect) and east-west (eastness; sine of aspect) components, since these better represent insolation. National Vegetation Information System (NVIS) vegetation categories were generated from NVIS Major Vegetation Subgroups data ( $n=38$ categories) (Version 4.1) (Department of Sustainability Environment Water Population and Communities, 2015) originally obtained at $100 \times 100 \mathrm{~m}$ resolution. We resampled this to $250 \times 250 \mathrm{~m}$, assigning the most common cell value to the $250 \mathrm{~m}$ cell. This resulted in 39 environmental variables.

Variables incorporated into SDMs should be ecologically relevant, and while correlations should be minimised, Maxent's predictions are considered robust to correlated variables (Elith et al. 2011; Guisan \& Zimmermann 2000). We developed competing models using different sets of environmental variables. The first model incorporated all 35 BIOCLIM covariates only, consistent with a previous SDM for the species (Claridge 2002). Four alternative subsets of the 39 variables were explored, informed by ecological relevance, expert feedback, variable contribution, and variable correlation. All models produced high AUC scores (> 0.9). The final 'pre-survey' model was selected based on visual inspection of the resulting map and guidance from potoroo experts. This model included the following seven variables: maximum temperature of the warmest week, precipitation of the driest week, highest weekly radiation, lowest weekly radiation, lowest weekly moisture index, mean moisture index of wettest quarter, and mean moisture index of driest quarter. These 
variables were selected due to their influence on the species' primary limiting factor, the availability of fungi. Hotter, drier conditions will negatively affect soil and vegetation characteristics and limit the presence, diversity and abundance of associated hypogeal fungi (Claridge et al. 2000). Two variables, lowest weekly moisture index (BIOCLIM 30) and mean moisture index of the wettest quarter (BIOCLIM 33) were highly correlated (Pearson's $r>0.8$ ) (Appendix S6), however, the model was regarded by experts to be the most reasonable representation of the long-footed potoroo's likely distribution. The models were fitted with each set of variables using five-fold cross validation to define training and test data sets and to enable evaluation of model performance using the Area Under the Receiver Operating Characteristic curve (AUC). Maxent was permitted to generate linear, quadratic, and product features (transformations of the provided predictor variables). A multivariate environmental similarity surface (i.e., MESS maps; Elith et al. 2010) was generated (Appendix S2), indicating areas that involved model extrapolation to environmental conditions beyond those represented in the model-fitting data.

\section{Background Points}

By default, Maxent randomly samples 10,000 points from across the study area (the "background") and compares the environmental characteristics at these points to the characteristics at occurrence points. This approach assumes that the occurrence records are also a random sample from suitable locations across the landscape (Elith et al. 2011). However, sampling bias (e.g., towards settlements and accessible areas) is likely to be present in the occurrence data because of the ad hoc nature of many of these collections. Accounting for sampling bias is critical to the accuracy of SDMs generated from presenceonly datasets, and failure to correct for it can result in predictions that reflect not just the distribution of suitable habitat but also of sampling effort (Phillips et al. 2009; Syfert et al. 2013; VanDerWal et al. 2009). Hence, to reduce sampling bias, we followed the approach outlined by Elith et al. (2011) by randomly selecting $\sim 10,000$ background records from all mammal records in the ALA that were recorded from the four bioregions (Interim Bioregionalisation of Australia, Thackway \& Cresswell, 1995) within which the long-footed potoroo occurs (Australian Alps, South East Coastal Plain, South East Corner, South Eastern Highlands). Sampling bias present in observations of these mammals will not perfectly reflect that for the long-footed potoroo, as there have been some strategic, planned surveys for this species. However, we expected that the bias would be captured more effectively than if Maxent's default, random background was used. 


\section{Long-footed potoroo Field Survey}

\section{Study Location}

203 The study area was located in South-East Forests National Park (SEFNP), in south204 eastern New South Wales (NSW) (Figure 1; Appendix S3). NSW survey sites for the 205 present study were located on seven vegetation classes: South East Dry Sclerophyll 206 Forests, Southern Hinterland Dry Sclerophyll Forests, Southern Escarpment Wet 207 Sclerophyll Forests, South Coast Wet Sclerophyll, Southern Warm Temperate Rainforests, 208 Tableland Clay Grassy Woodlands, and Eastern Riverine Forests (Office of Environment 209 and Heritage, 2012) (Appendix S4). Elevation across sites ranged from 170 to $1,144 \mathrm{~m}$ 210 a.s.I. The SEFNP is bounded predominantly by areas of State Forest and private 211 agricultural land-holdings.

\section{Site Selection}

214 Survey sites (i.e. $500 \mathrm{~m}$ transects) in NSW were selected based on Maxent's model output. To meet our dual objectives of species detection and ground truthing the model, sites were stratified across the predicted habitat suitability (HS) gradient. Model output (i.e. HS values) was reclassified into four climate suitability bands that were loosely based upon the range of predicted HS values at locations where long-footed potoroos had been detected previously:

We overlaid the reclassified map of the study area with topographic layers (rivers, contours, land-use types (SEFNP boundaries vs State Forest), and roads), and haphazardly selected 58 survey sites that were located within $100 \mathrm{~m}$ of the nearest access track and were primarily within SEFNP boundaries (thereby ensuring accessibility). In addition, as the long-footed potoroo's home range has been estimated to be between 14100ha (Green et al. 1998; DSE 2009), we ensured that sites were independent by

a) Low (Unsuitable): 0-0.13 (i.e. below the "equal test sensitivity and specificity" binary threshold of 0.13 , which was based on the average of five cross-validation folds);

b) Low-Moderate: $0.13-0.61$ (above "Low", but below the $50^{\text {th }}$ percentile HS value of known occurrences);

c) Moderate: $0.61-0.89$ (between the $50^{\text {th }}$ and $100^{\text {th }}$ percentile of known occurrences); and

d) High: 0.89-1 (above HS values of known occurrences)

separating them by approximately $1 \mathrm{~km}$ or more. This distance is consistent with the recent 
235 long-footed potoroo survey carried out in Victoria (pers. comm. Andy Murray, DELWP, 1 236 December 2016).

237 Note that in NSW, there were no grid cells in the study area that were classified as having 238 'High' HS. Rather, areas with the highest HS values in NSW were in the 'Moderate' HS 239 band. From this 'Moderate' region, we identified 15 sites that met the above criteria. Of the 240 remaining sites, four were intentionally located close to potoroo occurrence records in 241 NSW (within $~ 500 \mathrm{~m}$ ) ( $\mathrm{n}=3$ situated in 'Low-Moderate' HS band; and $\mathrm{n}=1$ in the 'Low' HS 242 band) and the rest were allocated approximately equally across the 'Low-Moderate' ( $n=$ $24320)$ and 'Low' $(n=19)$ HS bands (Figure 1; see also Appendix S3 for detailed maps). Most 244 sites (49) were purposefully located in areas that had not been logged or exposed to fire 245 for at least 25 years, giving sufficient time for forest succession to occur and resulting in 246 greater habitat complexity (Coops \& Catling 2000).

\section{NSW Survey: Camera Trapping}

248 Due to the likely low population density and patchy distribution of the long-footed potoroo 249 in NSW, individuals may be difficult to detect using traditional survey methods, e.g. live 250 trapping and hair tubing. As such, camera trapping is likely more effective (Scroggie et al. 251 2011), with recent camera trapping in Victoria proving highly successful for detecting the 252 potoroo (pers. comm. Andy Murray, 1 December 2016, and Marc Perri, Department 253 Environment Land Water \& Planning (DELWP), 6 December 2016).

254 We performed camera surveys in NSW during six field trips between April 2016 to May 255 2017. Up to 10 sites were trapped on each trip, and at each site 6 - 8 baited camera 256 stations were deployed along the $500 \mathrm{~m}$ transect, $100 \mathrm{~m}$ apart. Baits, containing oats and 257 peanut butter or peanut butter only, were suspended $20-30 \mathrm{~cm}$ above ground (Claridge et 258 al. 2016; Paull et al. 2011). Following Claridge et al. (2010) and Taylor et al. (2014) 259 cameras were mounted on a tree $(40-80 \mathrm{~cm}$ above the ground) at a distance of $1.5-2 \mathrm{~m}$ 260 from the bait station and left in-situ for up to 63 days (minimum 52 days; median of 59) 261 (Appendix S5). This extended deployment time, more than double that recommended for 262 95\% detection of the species (Scroggie et al. 2011), was considered necessary to 263 maximise detection probability.

264 Two types of passive infrared (PIR) cameras (four Reconyx Hyperfire HC600 (hereafter 265 "Reconyx") and up to four Scoutguard DTC-530V (hereafter "Scoutguard")) were placed 266 along each transect in a consistent order (i.e. two sites containing both cameras, two sites 267 with Reconyx only and two sites with Scoutguard only; however, when only six cameras 268 per site were available it was four sites with Reconyx only and two with Scoutguard only). 
269 The motion detection trigger settings were: i) Reconyx: "High" sensitivity, 5 images per 270 trigger event, 1 second delay between triggers and 1 minute quiet period; ii) Scoutguard: 271 "High" sensitivity, 3 images per trigger, 5M resolution (highest resolution setting) and 1 272 minute quiet period. Camera images were reviewed by MWD and Office of Environment 273 and Heritage $(\mathrm{OEH})$ experts. Images of potoroos were forwarded to two additional potoroo 274 experts for species identification and confirmation.

\section{Data Analysis}

277 Model validation with an independent dataset

278 Modelling species' distributions is an iterative process and models should be assessed 279 and recalibrated as new information becomes available. After completion of fieldwork, which was guided by the "pre-survey" SDM, three additional long-footed potoroo presence and absence datasets became available. These were: i) absence data generated from the current NSW field survey $(n=58)$; ii) presence data from Victorian camera trap surveys conducted during 2012-2016 ( $\mathrm{n}=91$ ), but not incorporated in the Victorian Biodiversity Atlas at the time of Maxent modelling (data courtesy of Marc Perri, 6 December 2016); and iii) absence data from Victorian camera trap surveys conducted in $2012(n=128)$ (data courtesy of Jenny Nelson, DELWP, 5 July 2017, refer to Lumsden et al. (2013) for further details on survey methods). Hence, these records constitute a dataset independent of that used in the original modelling.

289 To assess the predictive performance of the pre-survey SDM, we computed HS values for presence and absence records in the independent dataset, and evaluated whether the medians of these two groups differed, using a Kruskal-Wallis H Test (Bennett 1993) in Minitab® Statistical Software (Version 17) (Minitab Inc, 2014).

\section{Updated post-survey Maxent models}

New observations may help to refine estimates of a species' realised niche, or aid removal of bias (Guisan et al. 2006). The nature of available data at the time of initial modelling meant that the NSW field survey was informed by presence-only modelling techniques. Hence, an updated 'post-survey' Maxent model was fitted that incorporated an additional 54 long-footed potoroo presence records from Victoria, spanning the period 2012-2016, that were not previously included, giving a total of 395 presence records. The same Maxent settings, background locations, and pre-survey model covariates were applied. 
Raster Calculator tool in the ESRI ArcGIS software, ArcMap 10.5 304 (http://desktop.arcgis.com/en/arcmap/), which was used to compute a difference map 305 expressing the difference in HS values projected by the two models.

306

307

308

309

310

311

312

313

314

315

316

317

318

319

320

321

322

323

324

325

326

327

328

329

330

331

332

333

\section{Results}

\section{Pre-Survey: Maxent Species Distribution Model}

The pre-survey Maxent model had an average cross-validated test AUC value of 0.941 $(S D=0.011)$, indicating that the modelled outcomes could be considered a potentially useful prediction of habitat suitability (Phillips \& Dudík 2008). The most important variables were mean moisture index of the highest quarter (29.7\%), maximum temperature of warmest week (24\%) and mean moisture index of lowest quarter (19.9\%) (Appendix S6). It is noted, however, that while mean moisture index of the lowest quarter and lowest weekly moisture index each provided unique contributions to the model, they were highly correlated (Appendix S6), and thus the importance of the latter may be underestimated. Covariate response curves indicated that the highest predicted HS values are in areas with higher moisture (Appendix S6).

The model projected a large, contiguous area in Victoria to have the highest HS values, consistent with the locations of known populations (Figure 2). In comparison, the few areas in south-eastern NSW projected to have 'Moderate' HS are relatively small and fragmented (Figure 2). This finding is similar to the historical BIOCLIM output, which identified a large area of suitable habitat in East Gippsland, Victoria, and smaller patches in NSW (Claridge 2002).

Of the 34 potoroo records in NSW, all are located in the 'Low-Moderate' HS band; with many records located on flatter areas. As such, a number of 'Moderate' HS patches in NSW (e.g. around Candelo Creek, Back Creek, Basin Creek, and Reedy Creek) do not contain any known occurrences, and these may provide promising candidate locations for survey work, though some sites are relatively inaccessible (i.e. at higher elevations, steeper slopes or along the sides of ridgelines). Areas predicted to have 'Moderate' HS values occur within the SEFNP as well as State Forest, the latter of which is actively logged and hence may be too disturbed to support potoroos. 


\section{Camera Survey}

335 During this study, a total of 215,759 images (including false triggers) capturing 43 species 336 from 58 transect sites (or 431 individual camera sites) were recorded from 25,120 camera trap nights. Incorrect camera settings (e.g. time lapse / video) on four cameras generated a large number of images $(102,412)$, of which only night images were reviewed in detail. Images of potoroos were examined by experts (A. Claridge, A. Murray), who confirmed they were of the long-nosed potoroo (Potorous tridactylus).

341 Fifteen native mammal species, 22 bird species and one reptile species were detected. Seven introduced mammal species were also detected. Of the mammals identified, the long-nosed potoroo and southern brown bandicoot (Isoodon obesulus) are listed as 'Vulnerable' and 'Endangered', respectively, under the Environment Protection and Biodiversity Conservation Act 1999, and considered at risk of fox and cat predation. Of concern for native small- to medium-sized mammals is the dominance of foxes (Vulpes vulpes) and feral cats (Felis catus), detected at $28 \%$ and $43 \%$ of survey sites, respectively. Another potential predator, dingoes (Canis lupis dingo), were detected at $28 \%$ of sites. The prevalence of these predator species across survey locations is relatively high $(71 \%$ of sites), particularly considering that meat lures were not used, and sites were not situated on roads or tracks. A full list of the species detected, the total number of sites occupied and a breakdown across the Maxent model suitability bands is given in Appendix S7.

354 Despite our efforts, no long-footed potoroos were detected, hence survey sites in NSW were deemed 'absences'.

\section{SDM Ground Validation}

358 Using the independent datasets outlined above (see "Model Validation"), a comparison of HS values at presence $(n=91)$ and absence $(n=186)$ locations highlighted variability within these two groups as well as some overlap between them (Appendix S8). Furthermore, $72 \%$ of long-footed potoroo absences are in areas that may be considered "suitable" when the cross-validated mean 'equal test sensitivity and specificity' binary threshold is applied. Overall, however, the Kruskal-Wallis test of mean ranks revealed that 364 there is a statistically significant difference between HS values at presence and absence locations $(H=58.66, d f=1, p<0.01)$. This result suggests that the pre-survey Maxent model used to guide the field survey tends to perform well. 
369 The post-survey model generated an average cross-validated AUC of $0.944(\mathrm{SD}=0.007)$ 370 (slightly higher than the pre-survey model), suggesting that the model is a useful classifier 371 of habitat suitability (Phillips \& Dudík 2008). Maximum temperature of the warmest week 372 (27.4\%), mean moisture index of the lowest quarter (22.2\%), and mean moisture index of 373 the highest quarter (19.9\%) were found to contribute most to the fit of the model.

374 Comparison of the pre- and post-survey models revealed several regions where 375 projections of habitat suitability differed (Figure 3). In NSW, there are areas predicted to be relatively more suitable than indicated by the pre-survey model. It is unlikely that this would have given rise to significant changes to the selection of survey sites as, in general, accessible areas with the highest HS values in NSW were targeted, and these areas continue to have similar (i.e. 'Moderate') HS values in the updated model. Of interest, however, is that the interior Genoa area, north of Sheep Station Creek, and the Nungatta Plateau area (adjacent to Nungatta Station) are predicted to be relatively more suitable (as indicated in Figure 3). These areas are rather remote and difficult to access, hence were not targeted for our survey.

\section{The case of the missing potoroo}

Identifying where species occur underpins many conservation management decisions (Guisan et al. 2013) and the use of species distribution models to guide field surveys has been valuable for many species (Aizpurua et al. 2015; Guisan et al. 2006). However, no long-footed potoroos were detected during our SDM-guided surveys in NSW. As such, the distribution of this Critically Endangered species in NSW remains unclear and is founded upon records collected in the 1990s.

\section{Simply not there?}

394 We did not detect long-footed potoroos in any areas predicted to have relatively high habitat suitability (HS) values in NSW, or from a wider range of areas that were considered suitable when a binary threshold was applied to model output. Furthermore, the species was not detected at sites close $(<1 \mathrm{~km})$ to where historical records were obtained. 398 Possible explanations for the species' absence include: unsuitable microhabitat, fluctuations in population dynamics, impact of disturbance events (e.g. fire and logging) and presence of introduced predators and competitors. 
401 Microhabitat features are an important factor influencing the distribution of mycophagous 402 mammals, including long-nosed potoroos (Bennett 1993; Claridge \& Barry 2000; Vernes 403 2003) and it is possible that the resolution of the SDM (i.e. $250 \times 250 \mathrm{~m}$ ) did not capture 404 microhabitat characteristics. Generating predictive maps using SDMs requires covariate 405 datasets that span large spatial areas, which rarely exist for biotic and fine-scale abiotic 406 factors. We note, however, that a number of survey sites with 'Moderate' HS values 407 appeared visually to have appropriate microhabitat characteristics (e.g. Tantawangalo and 408 in pockets of temperate rainforest). In these locations, it is possible that the potoroo is 409 locally extinct, or was simply never there, particularly as many of these sites were distant 410 from historical presence records.

411 Some sites in NSW may have suitable microhabitat but are unoccupied because the patch 412 is either too small or located too far from populated sites. Indeed, most areas predicted to 413 have highest suitability in NSW were small and patchy (Figure 3). For instance, the area in 414 Tantawangalo with relatively high HS values was $\sim 950 \mathrm{ha}$ in size and located $\sim 10 \mathrm{~km}$ from 415 the next patch of suitable habitat at Wog Way, Coolungubra ( 780ha).

416 Habitat fragmentation is likely to exacerbate poor connectivity of climatically suitable 417 areas. There has been significant land use change in the study area over time, with large 418 areas of SEFNP bounded by pine plantations and pastoral lands. Fragmentation disrupts 419 ecological processes and restricts the movement of organisms, thereby isolating 420 subpopulations and increasing extinction risk (Gilbert-Norton et al. 2010; Worboys \& 421 Pulsford 2011).

422 Disturbance events such as logging and fire may also negatively impact the potoroo via 423 direct mortality, reduced vegetation cover and food availability, increased exposure to 424 predators, and disrupted social structure (DSE 2009). In an attempt to limit the influence of 425 these factors on detectability, most survey sites were situated in locations that had not 426 experienced fire or logging for more than 25 years. However, it was frequently apparent 427 that logging had occurred in the past, and may have contributed to absence or non428 detection of the species. We also note that many occurrence records are located in 429 previously logged forests (including the long-term Bellbird study site). This suggests that 430 regrowth forests are utilised by the potoroo (DSE 2009), although it is also known that 431 potoroos inhabiting mature, multi-aged and old growth forest tend to forage for shorter 432 periods, have smaller home ranges and are more fecund (Green \& Mitchell 1997; Green et 433 al. 1998). 
434 Finally, the presence of feral pigs (Sus scrofa), another highly mycophagous species, is a 435 key threatening process for the long-footed potoroo as both species compete directly for 436 hypogeal fungi (NPWS 2002). The camera survey detected feral pigs at approximately $43715 \%$ of sites. The long-footed potoroo is also at risk of predation by foxes and cats, which 438 were detected at nearly $50 \%$ of sites. These findings are of concern for the persistence of 439 the long-footed potoroo.

\section{A clever disappearing act?}

It is possible that the long-footed potoroo remained hidden, defying detection due to its likely low population density in NSW. In the present study we tried to maximise detection by using best practice survey methodologies: camera trapping, baited traps and extended deployment times. Camera trapping has been widely used for species surveys and has been more successful for detection of small- to medium-sized mammals, including the long-footed potoroo (Claridge et al. 2010; Paull et al. 2012; Smith \& Coulson 2012; Taylor et al. 2014), than other methods (e.g. Elliot traps, cage traps and artificial traps) (Welbourne et al.2015). Recent camera surveys for the species in Victoria utilised deployment times ranging between 21 days (Lumsden et al. 2013) and 35 days (pers. comms. Andy Murray, 1 December 2016), successfully recording the species at 41 (out of 170 ) and 35 sites (out of 85 ), respectively.

\section{Looking forward: ongoing model refinement}

The success of a field survey guided by an SDM hinges upon the quality of the underlying SDM. Guisan et al. (2006) noted four elements that contribute to the usefulness of a model: i) positional accuracy of species occurrence records; ii) inclusion of only ecologically relevant predictors that are expected to have a physiological effect on the species; iii) use of an appropriate model based on available data; and iv) generation of background points from presence sites of other species when absence data are not available. These elements were considered during the development of the Maxent model.

462 The choice of relevant predictors and appropriate resolution for data used in an SDM may invoke a trade-off between using larger, accessible datasets and spatial accuracy in describing a species-environment relationship (Le Lay et al. 2010). The Maxent model used here incorporated ecologically relevant climate variables that operate over larger scales. Independent model validation found that the pre-survey model could successfully 
distinguish between presences and absences, although there was variability of HS values within each group (i.e. presence/absence).

It is also worth noting that the NSW occurrence records were situated in areas predicted by the pre-survey model to have 'Low-Moderate' HS values, with the 'High' HS value areas predicted only in Victoria. This suggests that the climatic conditions in Victoria, where the majority of current occurrence records are situated, were less frequently encountered in SE NSW where only smaller, patchy areas of relatively higher suitability were predicted. The small number of occurrence records located in NSW makes it difficult to draw a conclusion about the veracity or validity of the model.

However, future models could potentially be refined by incorporating other relevant environmental predictors. For example, soil characteristics are likely to play an important role in predicting the presence of the potoroo by influencing vegetation type and soil moisture, both of which can impact fungal diversity and abundance. Structural attributes of the vegetation are also likely to be important. For instance, attributes of eucalypts (diameter at breast height and upper canopy cover) relate to availability of hosts for hypogeous fungi (Claridge et al. 2000), while understorey cover and density are important for providing shelter, nesting sites and protection from predators (Scotts \& Seebeck 1989; DSE 2009). As previously noted, connectivity and patch size could also be important predictors. Time and resource constraints limited our ability to include these variables in the pre-survey model, but consideration of these variables for inclusion in a future model would be highly worthwhile.

An alternate modelling approach, applied for another mycophagous species, the northern bettong (Bettongia tropica), was outlined by Bateman et al. (2012) who generated a resource SDM and a competitor SDM. The predicted values from these models were incorporated into an SDM of the target species (i.e. climate + resource + competition variables), giving rise to models with stronger evaluation measures (AUC) than models utilising climate data alone (Bateman et al. 2012). Such an approach may be useful for the long-footed potoroo, whose presence is likely limited by fungal availability, competitors and predators.

Finally, models incorporating both presence and absence data have been found to outperform presence-only techniques (Webb et al. 2014). Given the additional presence and absence data from camera surveys in Victoria from 2012-2016, as well as 'absence' data generated from our surveys, it would be worthwhile investigating alternative modelling 
500 techniques, e.g. regression based SDMs such as Generalised Linear Models (GLM) or 501 Generalised Additive Models (GAM).

502 The benefits of ongoing model refinement were highlighted by the post-survey Maxent 503 model. The inclusion of additional presence records from Victoria led to several areas 504 being identified as more suitable than previously predicted. These areas are relatively 505 isolated and inaccessible. Access to the Nungatta and Nalbaugh Plateaus via helicopter 506 was recently organised for OEH staff, and low-density camera surveys were undertaken. 507 This did not yield any new long-footed potoroo sightings (JB, unpublished data, November 508 2017). However, other nearby areas may be worth considering for future surveys. Prior to 509 conducting these surveys, the use of new data layers (e.g. vegetation structure estimated 510 by LiDAR) or technologies (e.g. drones) could be considered to identify suitable 511 microhabitat features that may improve the likelihood of the species being detected.

512 In conclusion, species distribution models provide a useful starting point for directing 513 limited resources toward field surveys for very rare and threatened species. The pre514 survey Maxent model presented in this paper predicted a distribution of habitat that 515 provided insights into a number of new survey locations for the long-footed potoroo in 516 NSW. Model validation using an independent presence/absence dataset supported the 517 predictive capabilities of the model. The field survey did not detect the species, although 518 there remain large areas of the SEFNP that were not surveyed, either due to time 519 constraints or limited accessibility. Areas closer to the coast (e.g. Nadgee Nature Reserve) 520 may also be worth surveying, particularly given recent findings of the species in coastal 521 habitats in Victoria. Model refinement that includes additional environmental factors, such 522 as soil type, biotic interactions, microhabitat features and connectivity, would be an 523 informative next step. Regardless, if the long-footed potoroo is still present in NSW, it is 524 likely to be extremely rare.

\section{Acknowledgements}

527 This project was conducted as part of a Master of Research (MRES) undertaken by 528 Mareshell Wauchope-Drumm at Macquarie University. The Department of Biology 529 contributed to the cost of fieldwork. The Office of Environment and Heritage (OEH) funded 530 Joss Bentley and provided fieldwork equipment. Franz Peters and Rachel Butterworth of 531 NSW National Parks and Wildlife Service are thanked for their invaluable guidance and 532 support in the field. 
All survey procedures were approved by OEH Animal Research Authority (approval number 150804/01) and Macquarie University Animal Ethics Committee (approval number 5201600599 - Collaboration (OEH 150804/01)).

\section{REFERENCES}

Aizpurua O., Cantú- Salazar L., San Martin G., Biver G., Brotons L. \& Titeux N. (2015) Reconciling expert judgement and habitat suitability models as tools for guiding sampling of threatened species. J. Appl. Ecol. 52, 1608-1616.

Bateman B. L., Vanderwal J., Williams S. E. \& Johnson C. N. (2012) Biotic interactions influence the projected distribution of a specialist mammal under climate change. Divers. Distrib. 18, 861-872.

Bennett A. (1993) Microhabitat use by the long-nosed potoroo, Potorous tridactylus, and other small mammals in remnant forest vegetation, south-western Victoria. Wildl. Res. 20, 267-285.

Chick R., Henry S., Kambouris P. \& Tennant P. (2006) The effects of timber harvesting on the long-footed potoroo (Potorous longipes). Parks and Forests Report Series, 061.

Claridge A. (2002) Use of bioclimatic analysis to direct survey effort for the long-footed potoroo (Potorous longipes), a rare forest-dwelling rat-kangaroo. Wildl. Res. 29, 193-202.

Claridge A. W. \& Barry S. C. (2000) Factors influencing the distribution of medium-sized ground-dwelling mammals in southeastern mainland Australia. Austral. Ecol. 25, 676-688.

Claridge A. W., Barry S. C., Cork S. J. \& Trappe J. M. (2000) Diversity and habitat relationships of hypogeous fungi. II. Factors influencing the occurrence and number of taxa. Biodivers. Conserv. 9, 175-199.

Claridge A. W., Paull D. J. \& Barry S. C. (2010) Detection of medium-sized grounddwelling mammals using infrared digital cameras: An alternative way forward? Aust. Mammal. 32, 165-171.

Claridge A. W., Paull D. J. \& Cunningham R. B. (2016) Oils ain't oils: Can truffle-infused food additives improve detection of rare and cryptic mycophagous mammals? Aust. Mammal. 38, 12-20.

Coops N. C. \& Catling P. C. (2000) Estimating forest habitat complexity in relation to time since fire. Austral. Ecol. 25, 344-351. 
Department of Sustainability and Environment (DSE) (2009) Action statement, Flora and Fauna Guarantee Act 1988 No.58 Long-footed potoroo (Potorous longipes). Victoria: Department of Sustainability and Environment, Victorian Government.

Department of Sustainability Environment Water Population and Communities (2015). Australia - Present Major Vegetation Subgroups - NVIS version 4.1 (Albers 100m analysis product). Accessed at http://www.environment.gov.au/erin/nvis/index.html on 17 August 2015.

Elith J., Graham C., Anderson R., Dudik M., Ferrier S., Guisan A., Hijmans R., Huettmann F., Leathwick J., Lehmann A., Li J., Lohmann L., Loiselle B., Manion G., Moritz C., Nakamura M., Nakazawa Y., Overton J., Peterson A., Phillips S., Richardson K., Scachetti-Pereira R., Schapire R., Soberón J., Williams S., Wisz M. \& Zimmermann N. (2006) Novel methods improve prediction of species distributions from occurrence data. Ecography 29, 129-151.

Elith, J., Kearney, M. \& Phillips, S.J. (2010) The art of modelling range-shifting species. Methods in Ecology and Evolution, 1, 330-342.

Elith J., Phillips S. J., Hastie T., Dudík M., Chee Y. E. \& Yates C. J. (2011) A statistical explanation of maxent for ecologists. Divers. Distrib. 17, 43-57.

Elsner W., Mitchell A. \& Fitzsimons J. (2012) Distribution of the long-footed potoroo (Potorous longipes) and the spot-tailed quoll (Dasyurus maculatus) in the Goolengook Forest, East Gippsland, Victoria. Aust. Mammal, 34, 100-107.

Evans J., Ji F., Lee C., Smith P., Argüeso D. \& Fita L. (2014) Design of a regional climate modelling projection ensemble experiment-NARCliM. Geoscientific Model Development. 7, 621-629.

Gilbert-Norton L., Wilson R., Stevens J. R. \& Beard K. H. (2010) A meta-analytic review of corridor effectiveness. Conserv. Biol. 24, 660-668.

Green K. \& Mitchell A. (1997) Breeding of the long-footed potoroo, Potorous longipes, (Marsupialia: Potoroidae), in the wild: Behaviour, births and juvenile independence. Aust. Mammal. 20, 1-8.

Green K., Mitchell A. \& Tennant P. (1998) Home range and microhabitat use by the longfooted potoroo, Potorous longipes. Wildl. Res. 25, 357-372.

Green K., Tory M., Mitchell A., Tennant P. \& May T. (1999) The diet of the long-footed potoroo (Potorous longipes). Austral. Ecol. 24, 151-156.

Greenberg J. \& Mattiuzzi M. (2015) Gdalutils: Wrappers for the geospatial data abstraction library (gdal) utilities. $\mathrm{R}$ package version 2.0.1. 
601 Groff, L. A., Marks, S. B., \& Hayes, M. P. (2014) Using ecological niche models to direct rare amphibian surveys: A case study using the Oregon spotted frog (Rana pretiosa). Herpetol Conserv Biol. 9, 354-368.

Guisan A., Broennimann O., Engler R., Vust M., Yoccoz N. G., Lehmann A. \& Zimmermann N. E. (2006) Using niche-based models to improve the sampling of rare species. Conserv. Biol. 20, 501-511.

Guisan A. \& Zimmermann N. E. (2000) Predictive habitat distribution models in ecology.

Hutchinson, M., \& Xu, T. (2015). Methodology for generating Australia-wide surfaces and associated grids for monthly mean daily maximum and minimum temperature, rainfall, pan evaporation and solar radiation for the periods 1990-2009, 2020-2039 and 2060-2079. NARCliM Report to the NSW Office of Environment and Heritage

Le Lay G., Engler R., Franc E. \& Guisan A. (2010) Prospective sampling based on model ensembles improves the detection of rare species. Ecography. 33, 1015-1027

Lumsden L., Nelson J., Todd C., Scroggie M., McNabb E., Raadik T., Smith S., Acevedo S., Cheers G. \& Jemison M. (2013) A new strategic approach to biodiversity management-research component. Arthur Rylah Institute for Environmental Research, Melbourne.

Merow C., Smith M. J. \& Silander J. A. (2013) A practical guide to maxent for modeling species' distributions: What it does, and why inputs and settings matter. Ecography. 36, 1058-1069.

Nix, H.A. (1986) A biogeographic analysis of Australian elapid snakes. Atlas of elapid snakes of Australia: Australian flora and fauna series 7 (ed. by R. Longmore), pp. 4-15. Bureau of Flora and Fauna, Canberra.

NSW National Parks and Wildlife Service (2002) Approved Recovery Plan for the Longfooted Potoroo (Potorous longipes). NSW National Parks and Wildlife Service, Hurstville NSW.

N.S.W. Scientific Committee (NSW SC) (2015) Final Determination - Long-footed \begin{tabular}{l} 
Potoroo, Office of Environment and Heritage. [Accessed \\
\hline
\end{tabular} 
http://www.environment.nsw.gov.au/resources/threatenedspecies/determinations/F DLongfootpotoCR\%20.pdf, 5 October 2015.].

Office of Environment and Heritage (OEH) (2012) Biometric vegetation types and endangered ecological communities of the Shoalhaven, Eurobodalla \& Bega Valley Local Government Areas. Technical Report. NSW Office of Environment \& Heritage, Queanbeyan.

Paull D. J., Claridge A. W. \& Barry S. C. (2011) There's no accounting for taste: Bait attractants and infrared digital cameras for detecting small to medium grounddwelling mammals. Wildl. Res. 38, 188-195.

Paull D. J., Claridge A. W. \& Cunningham R. B. (2012) Effective detection methods for medium-sized ground-dwelling mammals: A comparison between infrared digital cameras and hair tunnels. Wildl. Res. 39, 546-553.

Phillips S. J., Anderson R. P. \& Schapire R. E. (2006) Maximum entropy modeling of species geographic distributions. Ecol. Modell. 190, 231-259.

Phillips S. J. \& Dudík M. (2008) Modeling of species distributions with maxent: New extensions and a comprehensive evaluation. Ecography. 31, 161-175.

Phillips S. J., Dudík M., Elith J., Graham C. H., Lehmann A., Leathwick J. \& Ferrier S. (2009) Sample selection bias and presence-only distribution models: Implications for background and pseudo-absence data. Ecol. Appl. 19, 181-197.

R Development Core Team (2015) R: A language and environment for statistical computing. Version 3.1.1.

Rooper, C. N., Sigler, M. F., Goddard, P., Malecha, P., Towler, R., Williams, K., Wilborn, R., \& Zimmermann, M. (2016) Validation and improvement of species distribution models for structure-forming invertebrates in the eastern Bering Sea with an independent survey. Mar Ecol Prog Ser. 551, 117-130.

Saxon M. J. \& Noble, W. K. (1993) A report of intensive surveys for Potorous longipes conducted in south east New South Wales between August 1991 and 1992. Southern Region Threatened Species Research, April 1993.: NPWS, Heritage Assistance Scheme and Forestry Commission of NSW.

Scotts D. J. \& Seebeck J. H. (1989) Ecology of Potorous longipes (Marsupialia: Potoroidae), and preliminary recommendations for management of its habitat in Victoria: A report to the Australian National Parks and Wildlife Service. The Department.

Scroggie M., Henry S. \& Lumsden L. (2011) Approved survey standards: Long-footed potoroo Potorous longipes, version 1.0.). The Department of Sustainability and Environment. Available at 
672

673

674

675

676

677

678

679

680

681

682

683

684

685

686

687

688

689

690

691

692

693

694

695

696

697

698

699

700

701

702

703

704

705

706

http://www.depi.vic.gov.au/ data/assets/pdf file/0006/230838/7-Long-footed-

Potoroo-Survey-Standards-FINALv1.0 2MAY11.pdf [Accessed 30 August 2014].

Seebeck J. \& Johnston P. (1980) Potorous longipes (Marsupialia: Macropodidae); a New Species from Eastern Victoria. Aust. J. Zool., 28, 119-134.

Smith J. K. \& Coulson G. (2012) A comparison of vertical and horizontal camera trap orientations for detection of potoroos and bandicoots. Aust. Mammal. 34, 196-201.

Stratmann, T. S., Barrett, K., Floyd, T. M. (2016) Locating suitable habitat for a rare species: Evaluation of a species distribution model for bog turtles (Glyptemys muhlenbergii) in the southeastern united states. Herpetol Conserv Biol. 11, 199213.

Syfert M. M., Smith M. J. \& Coomes D. A. (2013) The effects of sampling bias and model complexity on the predictive performance of maxent species distribution models. PLOS ONE. 8, e55158.

Taylor B. D., Goldingay R. L. \& Lindsay J. M. (2014) Horizontal or vertical? Camera trap orientations and recording modes for detecting potoroos, bandicoots and pademelons. Aust. Mammal. 36, 60-66.

Thackway R., \& Cresswell I. D. (1995) An Interim Biogeographic Regionalisation for Australia: a framework for establishing the National System of Reserves, Version 4.0. Australian Nature Conservation Agency, Canberra, 88.

VanDerWal J., Shoo L. P., Graham C. \& Williams S. E. (2009) Selecting pseudo-absence data for presence-only distribution modeling: How far should you stray from what you know? Ecol. Modell. 220, 589-594.

Vernes K. (2003) Fine-scale habitat preferences and habitat partitioning by three mycophagous mammals in tropical wet sclerophyll forest, north-eastern Australia. Austral. Ecol. 28, 471-479.

Webb M. H., Wotherspoon S., Stojanovic D., Heinsohn R., Cunningham R., Bell P. \& Terauds A. (2014) Location matters: Using spatially explicit occupancy models to predict the distribution of the highly mobile, endangered swift parrot. Biol. Conserv. 176, 99-108.

Welbourne D. J., MacGregor C., Paull D. \& Lindenmayer D. B. (2015) The effectiveness and cost of camera traps for surveying small reptiles and critical weight range mammals: A comparison with labour-intensive complementary methods. Wildl. Res. 42, 414-425.

Woinarski J. \& Burbidge A. A. (2016) Potorous longipes. The IUCN red list of threatened species, 2016: [Online]. [Accessed e.T18102A21960440. 
Worboys G. \& Pulsford I. (2011) Connectivity conservation in Australian landscapes. Environment.

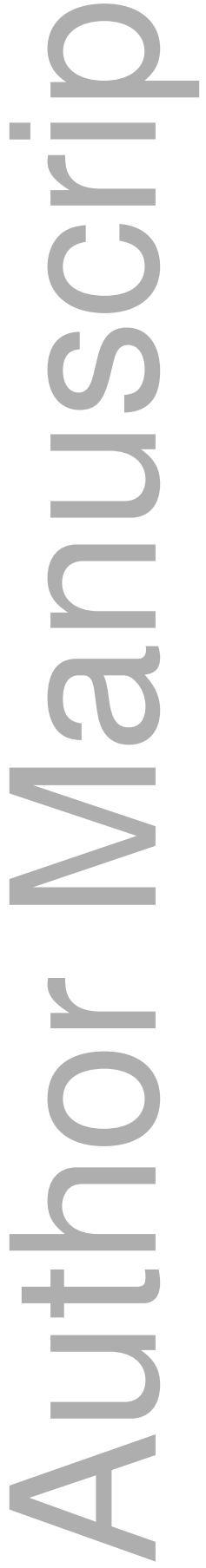




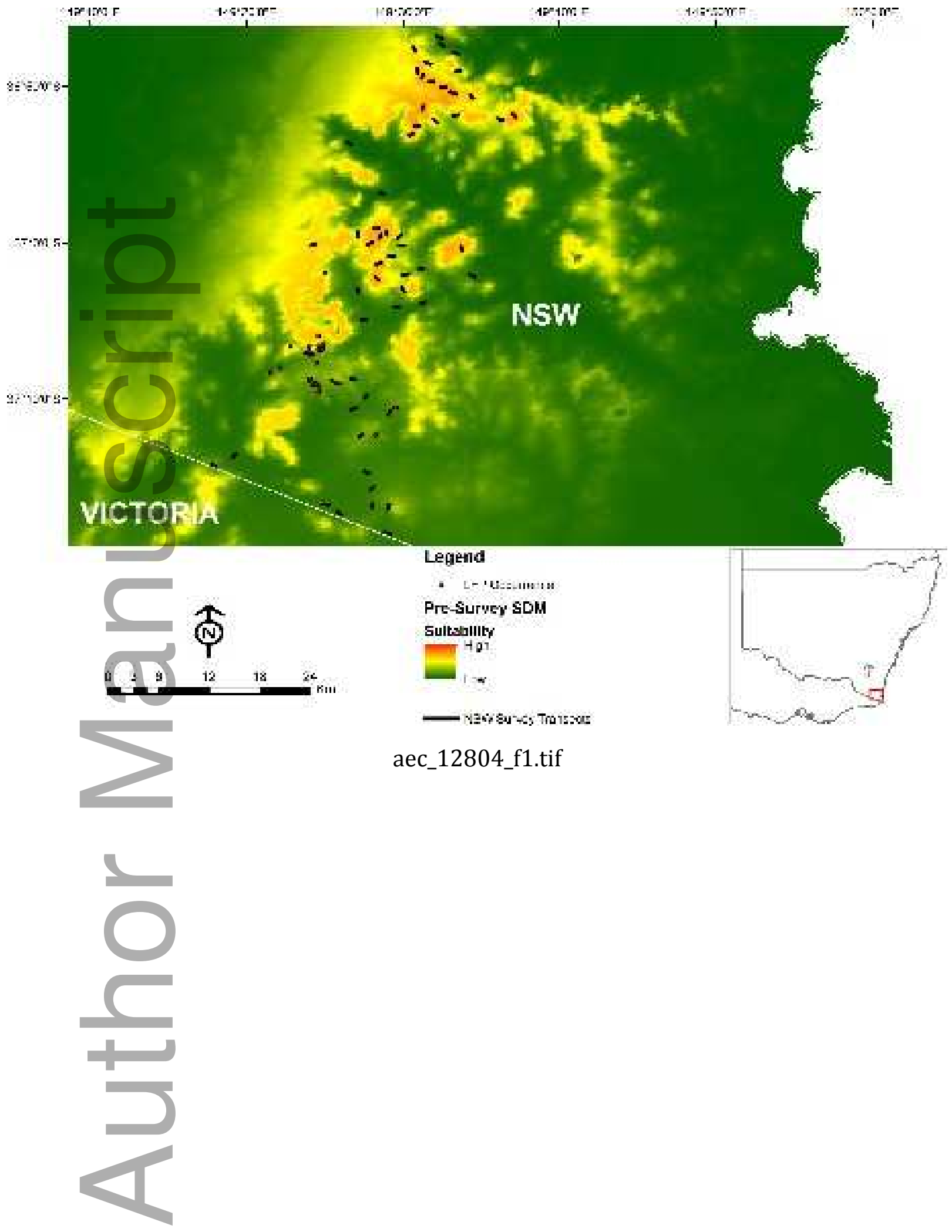

This article is protected by copyright. All rights reserved 


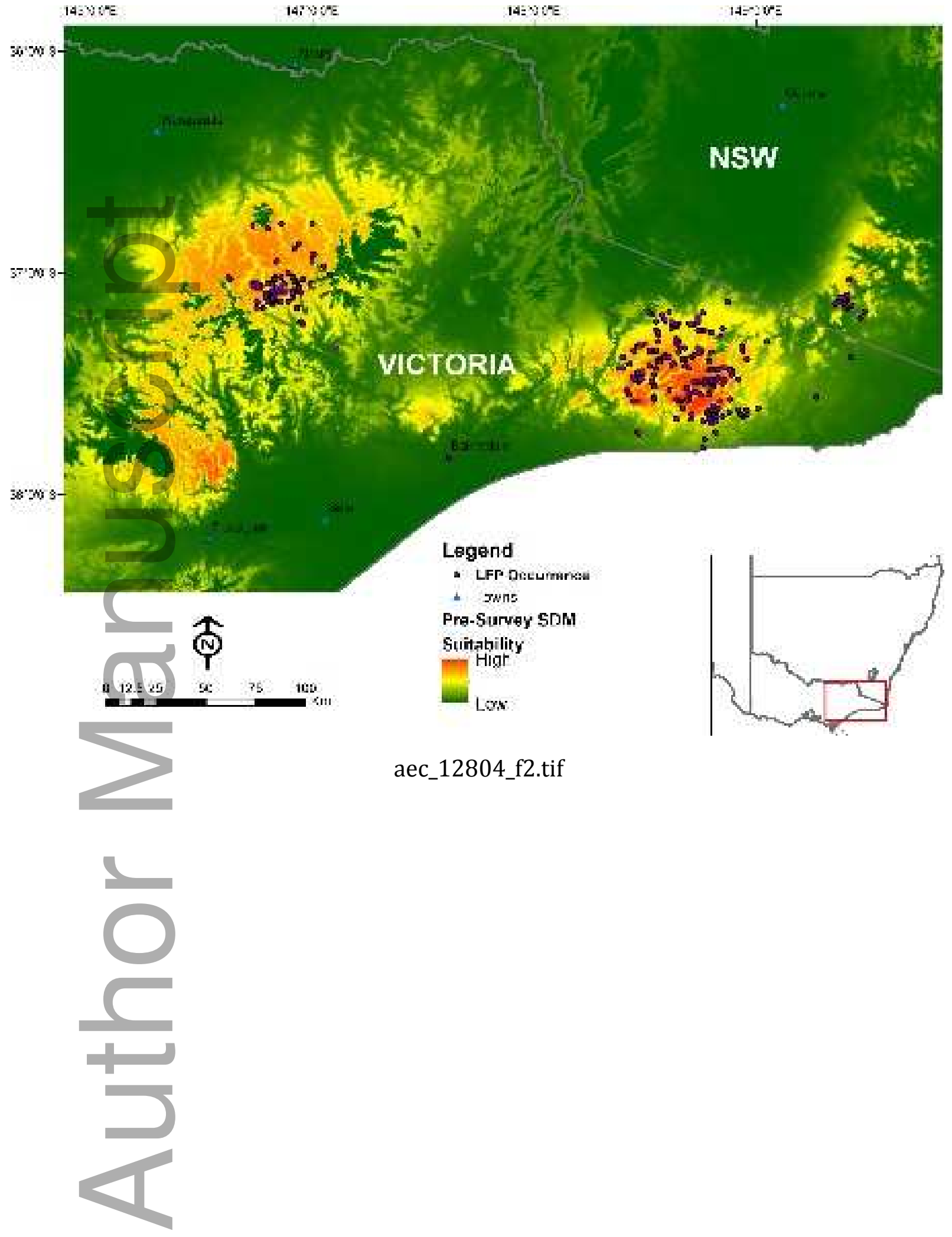

This article is protected by copyright. All rights reserved 


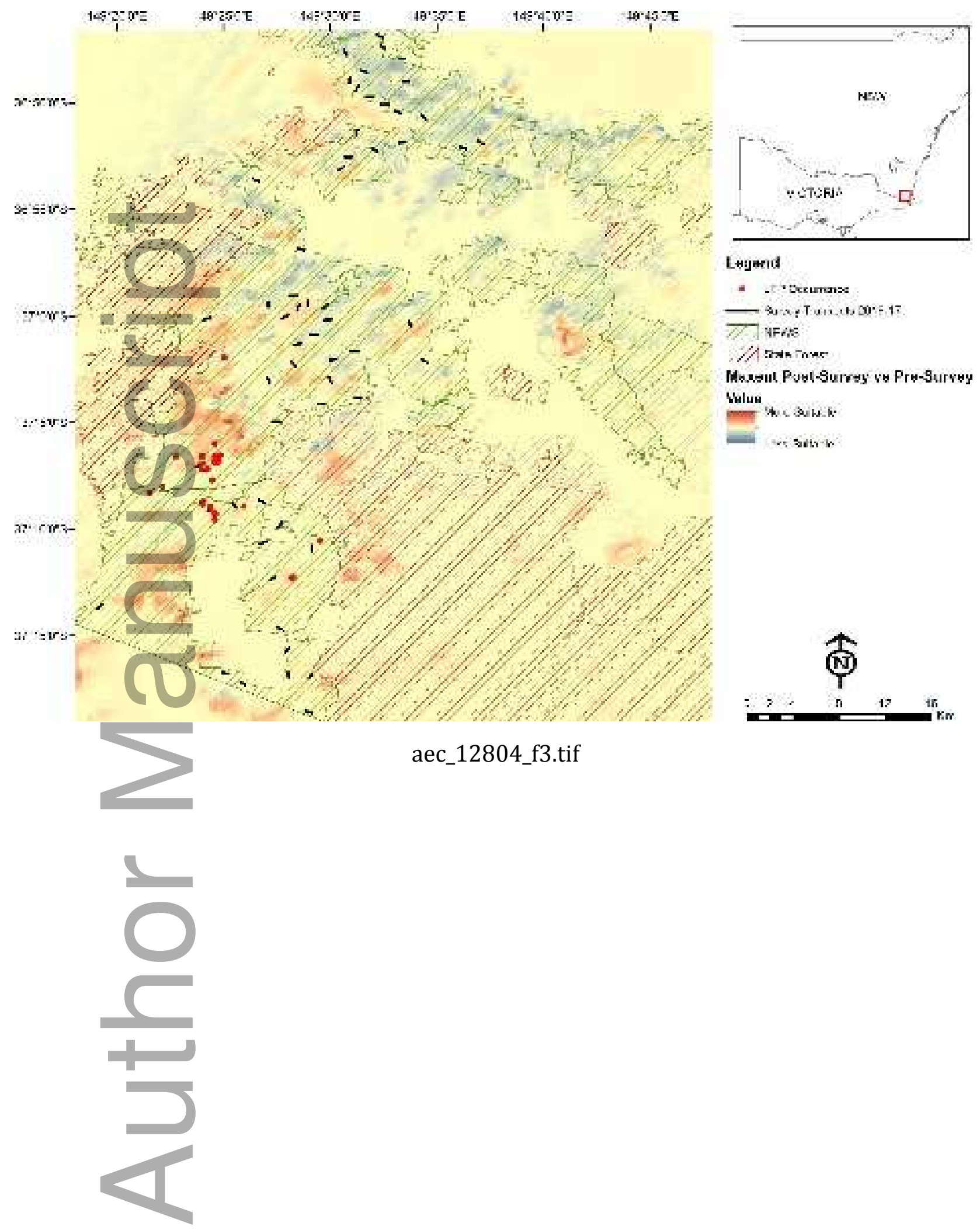

This article is protected by copyright. All rights reserved 


\section{University Library}

\section{- M M N E R VA A gateway to Melbourne's research publications}

Minerva Access is the Institutional Repository of The University of Melbourne

Author/s:

Wauchope-Drumm, M;Bentley, J;Beaumont, LJ;Baumgartner, JB;Nipperess, DA

Title:

Using a species distribution model to guide NSW surveys of the long-footed potoroo (Potorous longipes)

Date:

2019-10-30

\section{Citation:}

Wauchope-Drumm, M., Bentley, J., Beaumont, L. J., Baumgartner, J. B. \& Nipperess, D. A. (2019). Using a species distribution model to guide NSW surveys of the long-footed potoroo (Potorous longipes). AUSTRAL ECOLOGY, 45 (1), pp.15-26. https://doi.org/10.1111/ aec.12804.

Persistent Link:

http://hdl.handle.net/11343/286558 